\title{
Smart Perception System for SubSurface Robot Mapping: From Simulation to Actual System Realization
}

\author{
Ioannis Kostavelis, Dimitrios Giakoumis, \\ Evangelos Skartados, Andreas Kargakos, and Dimitrios Tzovaras \\ Centre for Research \& Technology Hellas, Information Technologies Institute, \\ 6th Km Charilaou-Thermi Road, 57001, Thermi-Thessaloniki, Greece \\ \{gkostave, dgiakoum, eskartad, akargakos, tzovaras\}@iti.gr
}

\begin{abstract}
Contemporary and anticipated future construction services give rise to an ever-increasing need for new technological, smart sensing solutions, which can give thrust in the automation of cross cutting applications with high societal and economic impact, including trenchless constructions, cabling and pipe installations, geotechnical investigations etc. In this line, this book chapter discusses the development of a smart perception system, composed of a Ground Penetrating Radar and a surface rover-like mobile robot, which is able to autonomously perform subsurface mapping. Firstly, the chapter presents a simulation tool which conceptually realizes the perception system by unifying surface exploration sensors with sensors used for subsurface modelling. This offers to the scientific community a valuable perception tool to perform simulation experiments on subsurface space. Secondly, the chapter documents the development of the actual smart perception system providing details regarding the integrated hardware as well as the developed methods that endow the system with autonomy features, which allow the systematic subsurface data collection, the automatic interpretation of the data for buried utilities detection, as well as their structured representation in the form of a subsurface map. The conceptual system evaluated on simulated environments, while the actual perception system evaluated on real test fields and both exhibited remarkable performance, proving thus their usability in the construction domain.
\end{abstract}

Keywords: subsurface mapping, simulation, surface rover, GPR

\section{Introduction}

There is a vast amount of applications which focus on the assessment of dense underground utilities in urban areas (e.g. electricity/communication cables, construction of pipelines, water and waste network), on the evaluation of the subsurface for energy and mineral production operations, as well as on the detection of buried objects for search and rescue applications at disaster sites. These applications require the modelling of the shallow (up to $5 \mathrm{~m}$ ) subsurface terrain, which is typically performed with specifically designed sensors namely, Ground Penetrating Radar (GPR) [1]. It is evident, that the technological achievements 
in the GPR imaging sensors witnessed a bloom during the last decades [2, 3], yet the existence of unified applications that allow smart and automatic representation of the subsurface area which can be commonly apprehended by humans and robots remains an open issue $[1,4]$.

Even if the biggest volume of GPR data processing has already been automated, still the experts' effort is required for further annotation to refine the initial findings, a factor that increases labor costs and withholds the analysis of large scale data back [5]. This relies on the fact that the acquired GRP data i.e. radargrams, are typically studied as isolated images for the detection of hyperbolic and linear patterns, that indicate the existence of an object, without the combination of multiple radargrams with their spatiotemporal extrapolation into the 3D space, where more comprehensive patterns can be revealed. Apparently, the lack of simulation environments that will facilitate the development of such methodologies without the need to have access in expensive GPR antennas and the absence of physically integrated subsurface scanning sensors with surface operating robots, prohibit the automated subsurface terrain modeling with cyber-physical systems.

Towards this direction, the goal of this chapter is two fold; One the one hand, it aims to introduce an integrated perception system in a simulation environment which is expected to offer roboticists with a valuable tool to perform simulations on subsurface area. To this end, the generated subsurface data obtained from the simulated perception system closely resemble the data obtained with real GPR systems allowing further integration and processing of the information regarding the buried objects in the subsurface. On the other hand, it documents a novel smart 3D underground mapping framework based on the integration of real GPR antenna with a surface operating rover, suitable for subsurface cyberphysical applications in robotics domain. This book chapter is grounded on our previous works for GPR simulation [6] and subsurface utility mapping [7], yet it has been extended to present more implementation details on the simulation environment, an exhaustive discussion of the radargram preprocessing steps as well as more evaluation results both in simulation and in real environment where the integrated smart perception system has been tested on a custom test field.

\section{Related Work}

\subsection{GPR Simulation tools}

Several tools such as the gprMax, the Reflexw, the GPRSim and other less common have been developed over the last years for the modelling and simulation of GPR. Reflexw covers the complete range of wave data such as seismic, GPR, ultrasound and the different geometry assemblies involving surface reflection and refraction, borehole crosshole and tomography and combination of borehole and surface measurements [8]. GPRSIM is an interactive 2D forward modelling software designed specifically for GPR and it can predict the full waveform of microwaves that are reflected, transmitted, refracted and attenuated across model ground structures [9]. The gprMax simulation tool, introduced by Warren et. al. 
[10] is the most prevalent simulation tool in the GPR community and comprises an open source software that contains a set of electromagnetic wave simulation tools based on Finite-Difference Time Domain numerical methods that offer many advanced features such as anisotropic and dispersive material modelling, realistic soil modelling, modelling of heterogeneous objects, objects with rough surfaces and modelling of GPR antennas. One of the biggest drawbacks, however, of the aforementioned simulation tools is that they are very costly both processing-wise and time-wise for the construction of a simulated environment of the required specifications. Moreover, they cannot be used for online simulations and neither can be integrated with other systems for joint simulation operations.

\subsection{GPR data pre-processing and hyperbola detection}

Authors in [11] presented a work for an image analysis technique capable of locating the position of the objects in the subsurface, by identifying the produced patterns on the GPR image. They grounded their technique on Hough Transforms and developed a method based on three parameters calculation, for each hyperbola found in the image. They were mostly focused on the modeling of the velocity propagation of the electromagnetic pulse in the material above the scattering object. This formatted the data to be analyzed on the field, allowing the scan to be repeated immediately when erratic results were obtained. The method has been applied on both synthetic and real data and revealed promising results. In another effort concentrated on the removal of the GPR data noise, the authors in [12] proposed a method to remove ringing noise that appeared as nearly horizontal and periodic event from GPR images. Specifically, the approach included a background removal with f-k filtering, predictive deconvolution with filtering in wavenumber domain, and filtering by Radon transform. It has been also shown that ringing can be successfully removed by the eigenimage filtering method, where GPR image is decomposed into eigenimages by singular value decomposition. Finally, it has been proven that these de-noising methods are more effective when compared with the simple methods for ringing noise removal, using realistic yet limited in number realistic images. Torrione et.al [13] proposed a method for landmine detection with GPR data, and they firstly proposed a pre-processing step using a computationally inexpensive algorithm that flags potential locations of interest. According to the authors, these flagged locations are then passed to a feature-based processer who further discriminates target-like anomalies from naturally occurring clutter. Specifically, the pre-processing step involved data aligning, ground-bounce removal and time clipping, media filtering to remove any GPS inference of the device and data depth-segmentation. The proposed method was applied on real data and allowed approximately $90 \%$ detection of buried utilities.

The first step towards underground mapping includes processing of the acquired GPR data for the detection of specific patterns such as hyperbolic or linear segments. The problem of hyperbola detection has been tackled by several approaches during the last years, the majority of which treat the problem with classical computer vision methods $[14,15,16]$. Specifically, among the 
most common techniques for hyperbola detection is the utilization of Hough transform, $[17,18]$ which takes advantage of the geometric characteristics of the primitive shapes that constitute a hyperbola signature. Yet, accurate capturing of the hyperbolas parameters with generalized Hough transform requires great discretization of its parameters, which increases significantly the computational time. A. Simi et.al [19] utilized Randomized Hough Transform while applying a threshold to candidate hyperbola apexes in order to extract hyperbola detections. A 2D map is produced by projecting the apex detections on a plane. Apart from hyperbolic patterns, Dell Acqua et al [20] developed a method for detection of linear objects (pipes) on GPR data based on Hough-Radon transform. The proposed algorithm was able to further analyse the data set in a local fashion, in order to eliminate spurious targets from the set of lines of maximum consensus. It has been evaluated on real data and proved adequate to detect pipes with an angular deviation in orientation of the linear segments less than 10o. As an extension of Hough-Transform method, Borgioli et al [21] introduced a weighting factor depending on the differentials of the unknown parameters with respect to the experimental errors, namely, the probe position error and the time-of-flight error. This feature enabled optimally placed sets of data pairs to be given greater weight than "ill-conditioned" sets, as for example when all data pairs lie near one end of the arc. The result is a decrease in the background amplitude with respect to the maximum of the peaks in the Hough accumulator space. It is shown that this improvement persists even when many arcs are present. Authors in [21] exploited a combination of Hough-Transform with Kirchhoff Migration to embed results from both techniques into a supervised framework to perform GPR data analysis. The evaluation was performed on 19 radar-grams and revealed accuracy in the detection of hyperbolic patterns above $90 \%$.

\subsection{Integration of GPR with surface operating rovers}

The existing technological background is focused mostly on the processing of $2 \mathrm{D}$ radar-grams (product of GPR imaging) in pixel-wise manner [22], while limited progress has been achieved in the construction of a global consistent 3D map of the subsurface environment. Moreover, even if the biggest amount of GPR data processing can be automated to an extent, still the opinion of experts for data annotation is required to reinforce the initial findings, a parameter that introduces extra labour costs and prohibits the large scale analysis of acquired data [5]. This is mainly due to the fact that the acquired radar-grams are mostly examined as isolated images for the detection of hyperbolic and linear patterns on them that indicate the existence of an object, without the combination of multiple scans with their spatiotemporal extrapolation into the 3D space, where more comprehensive patterns can be revealed. Synchronous arrays of GPR anten-

nas can provide massive subsurface data collection capabilities, and in order to achieve analogous large scale processping, the automation in structured acquisition procedures is imperative. The automatic data acquisition and the consistent processing of the all of the acquired GPR readings is feasible by incorporating the motion of GPR antenna into the processing loop. The latter necessitates 
GPR integration with a robotic carrier that would allow structured GPR motion modelling through the rigid body transformations of the robot. Such a topology has been realized in the work described in [23], where the authors developed a novel robotic system integrated with a GPR for automated bridge inspection. The collected GPR data were then analysed for the inspection of rebar used for the construction in the subsurface of a bridge. Although, the developed method proved capable of detecting defective spots in the subsurface of a bridge, the mobile robot was utilized only as the mean to carry the GPR antenna for the collection of the data while robots locomotion data were not considered during the process. The authors in [24,25] demonstrated an integrated robotic system that consisted of an all-terrain mobile robotic platform integrated with a GPR antenna and RGB cameras to collect GPR measurements that was intended to be utilized for surface and subsurface inspection of a bridge. In another application, the authors in [26] employed an all-terrain mobile platform to carry a GPR antenna, a system specifically hardened to operate in extreme temperatures of South Pole station of Antarctica. The objective of the mission was to gather subsurface data with the GPR for off-line inspection of ice sheets. It is revealed that a common characteristic among the above mentioned works is that the robotic topologies were designed to operate in relatively flat terrain where instantaneous changes of robot attitude were absent. Specific attempts have been put through in order to develop integrated robotic systems for subsurface inspection in uneven terrain such as the ExoMars mission which involved the development of the WISDOM Radar to be mounted on the ExoMars rover aiming to inspect the Mars subsurface and identify the best locations for drilling during its travel. The designed radar should provide information about the nature of the shallow subsurface over depth ranging from 3 to $10 \mathrm{~m}$ [27]. The interpretation of GPR data depends greatly on knowledge of the GPR sensors spatial orientation and relation to surface features, and, thus, it is essential that any autonomous GPR study have such a capability. Several advances in the automation of GPR data acquisition have used Differential GPS (DGPS) [28, 29] a theodolite based on laser, or proprioception to track the pose of the GPR during data collection . Barfoot et. al. answered to the above limitations by integrating for the first time a GPR antenna with a robotic platform through visual odometry (VO)[30]. The resulting system was capable of providing a three-dimensional, photorealistic surface model coupled with a ribbon of GPR data, and a two-dimensional, topography corrected GPR radar-gram with the surface topography plotted above on it. The outcome was an autonomously generated coupled surface/subsurface representation, however, a 3D metric map of the subsurface was not provided by that method.

\section{GPR Imaging}

To achieve large scale automated data acquisition of subsurface data, the GPR sensor is typically integrated with wheeled mobile vehicles. In our approach we assumed the existence of a GPR antenna which is an array of GPR sensors placed 
on specific rigid topology. Moreover, the GPR antenna is towed by a wheeled mobile vehicle to allow coverage of long distances and, thus, to facilitate large subsurface perception capacity. The working principal of GPR is relied on the transmission of electromagnetic pulses into the ground at frequencies usually in the range of 10-4000 MHz, depending on the design of the device and the target application [1]. During the signal travel in the subsurface, when it encounters an interface between layers of differing permittivity, part of the energy is reflected back to the surface while the remainder is diffracted from the subjacent medium. The reflection/diffraction process continues until the signal has weakened completely or the amount of time that the GPR receiver is programmed to search for a return signal (design principal of GPR device) has passed

\subsection{Perception with the simulation tool}

As mentioned in the previous sections, several tools such as gprMax, Reflexw, GPRSim etc. have been developed over the last years for the modelling and simulation of GPR. One of the biggest drawbacks, however, of the aforementioned simulation tools is that they are very costly both processing wise and time-wise for the construction of a simulated environment of the required specifications. Moreover, they cannot be used for online simulations and neither can be integrated with other systems for joint simulation operations.

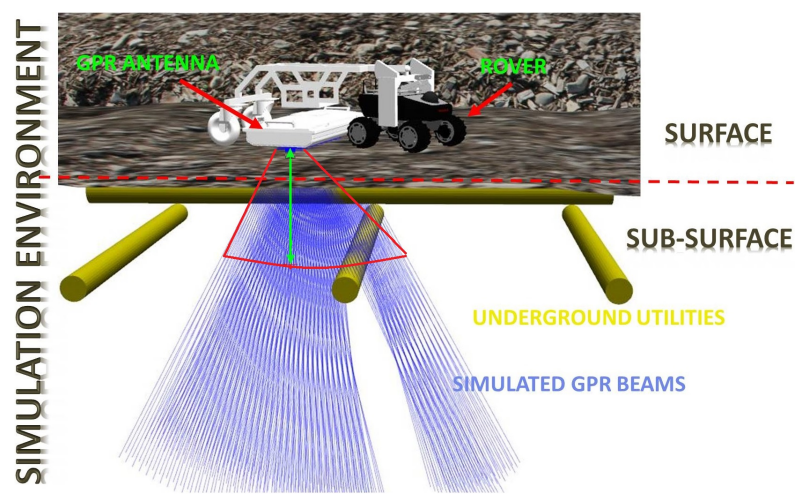

(a) Simulated GPR

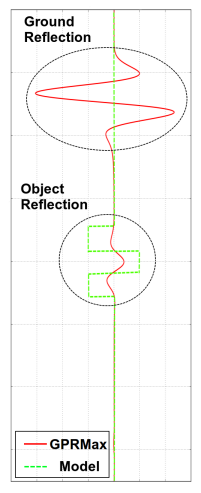

(b) Sample A-Scans

Fig. 1. Demonstration of simulated GPR Model. The range is extracted from ray casting all rays of the sensor and keeping the minimum range as depicted in 1(a) by the green dimension line. Sample A-Scans from gprMax and the proposed GPR Model are displayed in $1(\mathrm{~b})$

The proposed solution for the real-time simulation of GPR involves the utilization of sonar sensors. The operation of sonars is closely related to the operation of GPR antennas, with the main difference that sonars rely on sonic wave 
propagation in free space, while GPRs relies on electromagnetic wave propagation in the medium (ground). In robotics, simple low cost sonar sensors are widely employed to detect obstacles in the working environment of the robot. These sensors emit directed ultrasonic pulses that propagate in free space and record the time that the first reflection is received. This recorded time is then used to calculate the distance of the sensor to the closest object-obstacle that reflected the signal. Similarly, GPR antennas emit electromagnetic pulses that propagate in the subsurface. When these pulses meet a buried object or boundaries between materials of different permittivities, they can be reflected back to the surface where a receiving antenna records them for a fixed time window, resulting in a signal known as A-Scan. The appearance of a pulse in the A-Scan that closely resembles the emitted pulse, indicates the existence of an object or material boundary, the distance to which can be calculated provided the velocity in the propagation medium. Based on the above descriptions, it is apparent that sonars can be utilized for the approximate simulation of GPR antennas, with the assumption that the simulated subsurface environment is modelled as free space with various objects that will act as targets to be detected. The approach followed herein involves the utilization of array of GPR sensors simulated as sonars directed towards the ground and configured with minimum sensor range that surpasses the ground as shown in Fig 1(a). At the same time by exploiting a simulated world in which the ground is modelled as a plane with negligible depth and mostly empty space underneath, the sonar can sense whatever lies underground given that it is within its range. Due to the limitations of the utilized 3D simulator Gazebo ${ }^{1}$, the sonar sensors are simulated using a number of laser rays that form a $2 \mathrm{D}$ cone $^{2}$. Then, the desired range or the distance to the closest object in the ground is estimated by using ray casting for all rays of a sonar sensor and keeping the minimum resulting distance, as depicted in Fig. 1(b).

\subsection{Real smart perception system}

The real smart perception system composed by a commercially available surface operating rover and a GPR antenna. The selected rover is the Summit XL HL skid steering mobile robot paltform with payload capacity up to $65 \mathrm{kgs}$, suitable for navigation in uneven terrain and the towing of the GPR antenna. The utilized GPR is the Stream-C array provided by IDS GeoRadar. The central operation frequency of the antenna is about $600 \mathrm{MHz}$ and its typical depth range reaches $1.5 \mathrm{~m}$, providing an in-range resolution approximately $5 \mathrm{~cm}$. The system collects up to 32 radar profiles, having the antenna box a length of about $1.2 \mathrm{~m}$ and a weight around $20 \mathrm{Kg}$. The Stream-C with the Summit XL HL communicates with TCP/IP communication protocol for data transmission, which can be transmitted to any personalized devices enabling thus cyber-physical access to the system. Data acquisition and triggering from the GPR is performed through a programmable encoder wheel which has been mechanically integrated with the

\footnotetext{
${ }^{1}$ http://gazebosim.org/

2 http://wiki.ros.org/hector_gazebo_plugins
} 
left-back wheel of the rover so as the measurements to be obtained only when the robot is on move. The sampling rate of the GPR encoder has been set to $3 \mathrm{~cm}$, i.e. when the robot traverses $3 \mathrm{~cm}$, a trigger is activated and synchronous measurements from all the channels of the GPR are acquired. The electromechanically integrated surface rover is graphically illustrated in Fig. 2.

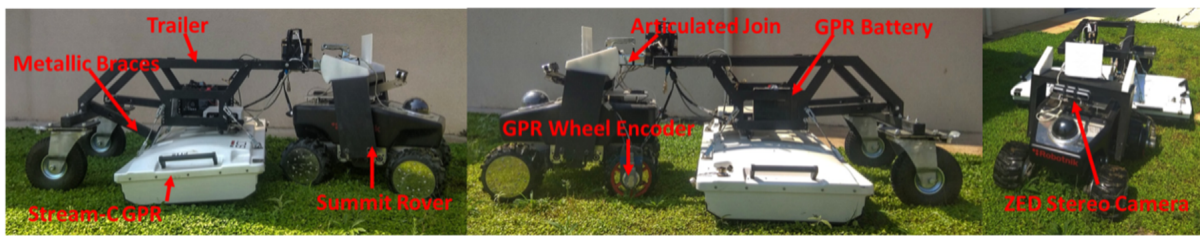

Fig. 2. The perception system constituted from the Summit XL HL Rover and StreamC GPR antenna

\subsection{B-Scan formulation}

Assuming that the robot travels on straight routes, each A-Scan corresponds to subsurface representation for a specific robot traversed distance i.e. $3 \mathrm{~cm}$ in our case. A single measurement from the GPR (single A-Scan) can provide information about the distance to the closest object that falls within the field of view of the sensor. However, this information is not enough for the estimation of the position of buried objects. This problem can be solved by migrating from one dimension to two dimensions which can be accomplished by taking multiple measurements along the robots direction. This operation results in the formulation of a B-Scan or a sequence of concatenated A-Scans, which means that by concatenating continuously obtained A-Scans, a B-Scan is progressively built. Each B-Scan corresponds to a vertical slice of the ground above which the GPR has passed, and is typically represented as an image, where the pixel intensities on the B-Scan correspond to the amplitude values of the A-Scan. For each sensor existing in the Stream-C GPR array or each sonar sensor in the simulation GPR tool, a separate B-Scan is constructed during robot motion. An illustrative representation of this procedure is described in Fig. 3(a). The formulated B-Scans, also known as radar-grams are then further processed for the detection of buried utilities. The existence of an object, e.g. pipe, beneath the trajectory of the GPR results in the formulation of a down facing hyperbola in the resulting B-Scan and by employing simple geometric analysis it can be derived that the apex of the hyperbola corresponds to the actual depth of the buried object. Fig. 3(b) illustrates how hyperbolas are formed given the aggregated data (B-Scans) during the robots motion in specific direction. Note that $X_{i-n}, X_{i}, X_{i+n}$, represent the rovers position in distinct time instances and $Z_{i-n}, Z_{i}, Z_{i+n}$ correspond to the respective closest depths recorded by the signal reflection. 


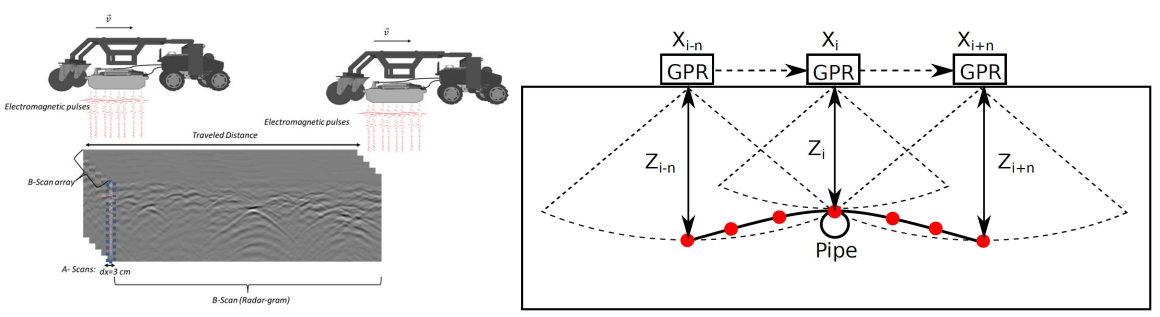

(a)

(b)

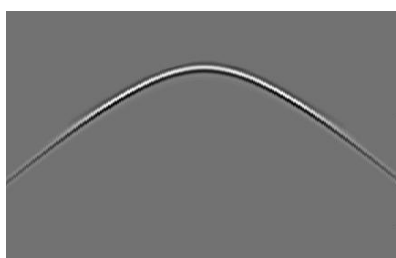

(c)

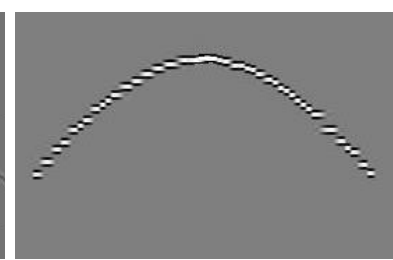

(d)

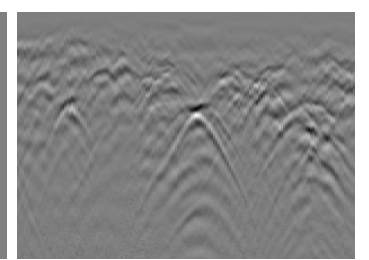

(e)

Fig. 3. On the upper row the B-Scan assembly is represented (left) and the hyperbola formulation given a traversal scanning of a pipe is illustrated (right). On the bottom row B-Scan samples with three different data acquisition sources are illustrated. From left to right, a) B-Scan from gprMax, b) B-Scan from proposed simulation GPR Model and c) B-Scan with the a real GPR antenna corresponding to a real subsurface environment

\subsection{Hyperbola detection in simulated B-Scans}

The hyperbola signature created from the simulated GPR (see Fig. 3(d)) tool is clear and noise-free mainly due to the fact that the subsurface modeled area is empty and there is now artifacts from the medium transmission of the emitted signal. Thus, for the detection of its apex a geometrical fitting method proved to be adequate. To this end, a simple hyperbola detection algorithm is applied, that is suitable for the GPR data generated using the proposed sonar-based simulated model. Specifically, the hyperbola detection algorithm considers a BScan and converts it to a normalized image. A preprocessing thresholding step aims to remove all pixels that do not belong to the hyperbolas. By applying the Hough transform for lines detection, the algorithm isolates and later removes all the detected straight lines that originate from pipes parallel to the direction of motion of the GPR. In the next step, the method applies a closing morphological transform [31], which is basically a dilation followed by an erosion operation that fills the gaps and completes the hyperbolic curves. The next step, involves the vertical averaging of the pixels to get a thinning of the hyperbolic curves, which are then fed to the well known DBSCAN [32] density-based clustering algorithm in order to segment the capable of segmenting the hyperbolas. The final step of the method comprises a smoothing via linear interpolation based on which the apexes of the hyperbolas can be isolated by simply finding the global maxima for each segmented hyperbola. 


\subsection{Hyperbola detection in real B-Scans}

Detection of hyperbola signatures in real B-Scans (see Fig. 3(e)) is a more challenging task, necessitating specific pre-processing steps in order to remove the noise and the ground bounce revealed from the alternation of the propagation medium (air-soil).

Pre-processing steps of real GPR data Considering the necessity of the preprocessing steps, a raw A-Scan captured from a GPR device typically suffer from two factors that negatively affect any further interpretation: DC bias and the "wow" effect. The first term describes a signal that has a mean value larger than zero, while the second one refers to a low-frequency trend in a recorded trace. Both of them are known to inherently exist in real GPR data, owing to the phenomenon of signal saturation by early arrivals, such as the ground/air wave, as well as inductive underground coupling effects. As a result, the spectrum of the recorded data is distorted and the performance of any technique based on spectral analysis deteriorates. Thus, a dewowing filtering is applied in accordance to which the DC component is removed by calculating the mean value of the single A-Scan trace and subtracting it from the signal values. Then, the removal of the low frequency component is applied by utilizing either a low-cut filter or equivalently a median filter. Another pre-processing step essential for the collation of A-Scans during antennas traverse is the Time Zero Correction. This is emerged by the fact that the exact time that the transmitted signal meets the ground varies even among consecutively captured measurements, and thus, cannot be predetermined since it is affected by a number of factors, including thermal drift, electronic instability and most importantly the spatial distance between the antenna and the ground. Therefore, the procedure of stitching together consecutive, individual A-Scans in order to formulate a B-Scan signal introduces a factor that could possibly lead to poor data quality. Through BScan generation we aim to extract an "image" of the underground and it only makes sense that consecutive samples that form this image should have a common origin that defines the point zero of the considered coordinate system. Since this common origin cannot be beforehand and explicitly determined in the time axis, it is necessary to define it through the recorded values. In order to achieve this uniformity in the constructed B-Scans, for all captured A-Scans the peak of the recorded signal which corresponds to the same event in time is detected and utilized to align those scans. All later events depicted in the assembly of the A-Scans will be correctly expressed with respect to a common origin. In Fig. 4(a) on the left image the peaks of adjacent A-Scans forming a B-Scan are annotated with red colour. The misalignment of those peaks introduces further artifacts on the time sequence of later events. On the middle image the Time Zero is adjusted for all Scans and artifacts are reduced. Finally, on the right image all values before Time Zero are discarded and the peak value is translated to the $t=0$ time sample.

Having formulating the B-Scan, the next step comprises the filtering of its content. The nature of the transmitted wavelet, which is prone to attenuation 


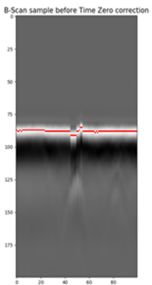

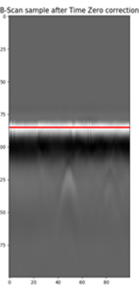

(a)

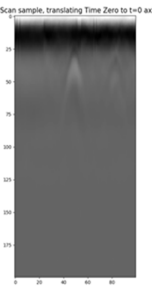

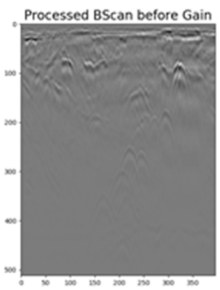

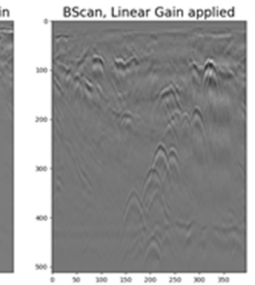

(b)

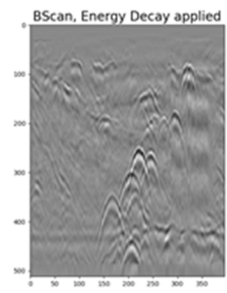

Fig. 4. On the left, an illustrative example of the time-zero correction pre-processing step; on the right, an example of the gain function filtering step in a B-Scan image

and dispersion effects, combined with the complex, cluttered and heterogeneous environments that are usually dealt with, the inherent system noise and finally the noise introduced from other outer sources (e.g. surrounding mobile and WiFi networks) make GPR data processing and interpretation a rather challenging task. Noise filtering is therefore a necessary pre-processing step towards gaining automatically understanding from GPR data. Temporal/Frequency filtering refers to the filtering of individual A-Scans in the time/frequency domain. One of the most important parameters characterizing a scanning procedure and dictating in particular the denoising procedure is the frequency of the transmitted pulse. The most appropriate type of filtering that should be applied on the individual GPR traces, for the purpose of rejecting out parts of the signal corresponding to irrelevant frequencies, is a bandpass filter and, thus, a Butterworth filter is utilized to take advantage of its properties in the bandpass zone. Spatial Filtering refers to the denoising techniques applied along the space axis (i.e. across the recorded values of all the A-Scan traces at a specific time). For the task of hyperbola detection the most appropriate spatial filtering, due to its properties is the Background Removal. The mean of the recorded amplitude is calculated across all A-Scans for each time value and is subtracted by them. In that way the removal of the ground-bounce is achieved and the effects of highly attenuating ground types (e.g. wet soil creating vertical signatures) are reduced. At the same time the hyperbola patterns are amplified. The last pre-processing step of the B-Scan is the Gain function which improves the visual representation of the information available in the processed GPR data. The gain function is achieved by multiplying all signal values with appropriate time varying factors. The calculation of those factors may take the physical process of the wave propagation into consideration. The energy decay cost function considers the wave losses to be connected to the travelled distance $d$ in a $1 / d 2$ manner and calculates coefficients based on the squared distance from the antenna. In fact, a linear, a polynomial, an exponential, or any kind of function connecting the travelled distance with some multiplication coefficients performing some kind of equalization can be utilized as a gain function. The results of a linear gain function and an energy decay function on a processed B-Scan are illustrated Fig. 4(b). 
Hyperbola detection on real GPR data Pre-processed A-Scans consist of consecutive positive and negative pulses, oscillating around a mean zero value. The hyperbola traces, which may indicate the existence of buried infrastructure, are expected to be shaped by either the positive or the negative pulses of consecutive A-Scans. The necessary positive-negative segmentation is performed by isolating sign changes on the A-Scan values around zero crossing. A zero crossing isolation step is applied to all A-Scans recorded in a scanning operation and a negative-positive separation on B-Scan, is produced. The isolation step is accompanied by a key point extraction method which is the location on the B-Scan image of the peak values of segmented pulses ( $\max$ and min values for positive and negative pulses respectively). This procedure produces the key-points which are nominal apexes of the existing hyperbolas in the entire radar-gram. The detected key-points are further utilized to produce 2D segmentations on the B-Scan. Specifically, each key-point constitutes a candidate apex and segmentation favoring hyperbolic shapes are performed around it. Two segmentation approaches are applied, both of which utilize data driven parameters regarding the maximum expected size of a single hyperbolic signature. The first segmentation merges into a hyperbola candidate all those pulses in the neighboring A-Scans of the candidate apex that have key-points that occur at the same or later time as the candidate one. The second segmentation step imposes a much stricter criterion that forces each new pulse to have a key-point value at a time strictly larger than the previous one. The first step is highly susceptible to noise pulses around the actual hyperbolic signature; however it generates candidates with slow increase of reception time amongst consecutive scans. The second step demands an increasing slope of at least 45 degrees asking from the reception time to constantly increase amongst consecutive scans distancing from the candidate apex. Consequently, the first segmentation produces better separation close to the hyperbola apex regions while the second segmentation performs better when distancing from the apex as hyperbolas slope converges to its asymptotes slope.

The next step comprises a feature extraction procedure based on Histogram of Oriented Gaussians (HoG), which instead of a fixed grid on the image plane, a grid with varying size is applied to each candidate apex on the produced segmentation. The applied patch size is related to the expected size of the hyperbola signature and is initially considered to be $30 \times 50$ pixels. On that patch a $6 \times 5$ cell grid composed of $5 \times 10$ cells is applied. For each cell the histogram of the gradients of the cell pixels contains 9 bins. The resulting feature vector length is equal to numberofcells $\times$ binsize $=270$. The basic advantage of the adopted solution is that each hyperbola is adaptively described by the feature vector depending on its size in the B-Scan. The extracted feature vectors are used to train a four class SVM classifier on which three classes are referred as positive ones that contain hyperbola traces and the negative class is referred to patches containing noise. The hyperbola detection considered as a classification task that has big intraclass variability due to the varying curvature that affects the shape and the final feature representation. Three separate positive classes of hyperbolas have been defined, according to the asymptotes slope, namely $22,5^{\circ}, 45^{\circ}$ and $67,5^{\circ}$ de- 
grees. During training of SVMs with one-vs-one approach, hyperbolic segments are clustered into the three positive classes, while noisy segments from real GPR images were used to define negative classes. During testing, comparisons are performed between all positive and negative classes and the one receiving the majority of votes is assigned to the segment under question. The classification step defines segments on the B-Scan positively identified as hyperbolas. The keypoint that produced the specific feature vector is not always sufficiently good location estimation on the radar-gram of the actual hyperbola apex and, thus, a hyperbola fitting step is performed after classification, where a gradient descent is applied to define the exact apex and curvature of the key-points of each detected patch that corresponds to a candidate hyperbola positively classified in the SVM. A hard threshold on the Least Square Error criterion applied on the fitting is used to further reject false positive hyperbolas.

\section{Subsurface mapping}

The next after the detection of the hyperbola apexes comprises the construction of the subsurface map, which is common both for the simulated as well as the real GPR images. To achieve this, localization information of the motion of the GPR antenna are required and, since the GPR antenna is towered by a surface operating rover, equipped with vision sensors, the robot's motion estimation is exploited. The GPR-rover topology is known and, thus, the surface rover motion estimation is inherited to the GPR antenna. As a result, the detected apexes, are expressed in global coordinates through the construction of subsurface map, endorsing the smart perception system with autonomy and large-subsurface mapping capacity.

\subsection{Surface rover localization}

For the robot motion estimation, a visual odometry algorithm has been implemented suitable to operate in outdoors environments. In particular a stereo camera mounted on the surface rover to perform incremental motion estimation based on visual features tracking, has been utilized. In each iteration loop a disparity image is computed through a stereo correspondence algorithm based on local block matching accompanied with global disparity space optimization [33]. The calculated disparity image is utilized to obtain the depth value $z(x, y)$ coordinates calculated through triangulation for each pixel $(x, y)$ of the left camera image, kept as reference frame as follows:

$$
z(x, y)=\frac{f \cdot B}{\operatorname{disp}(x, y)}
$$

where $f$ is the camera focal length, $B$ is there stereo camera baseline and $\operatorname{disp}(x, y)$. By applying the same procedure on all the pixels of stereo pair a dense 3D point cloud of the scene is obtained. However, for rough motion estimation between the consecutive frames, only a subset of the initial point clouds 
is required. In particular, among the potential detectors suitable for localization, the proposed motion estimation system employs Speed Up Robust Features (SURF) [34], which detects and matches the most prominent 2D points within two consecutive frames. The main reason for the utilization of SURF is its fast computation time compared to the existing methods. By evaluating the depth information at the respective $2 \mathrm{D}$ images, we obtain a set of point-wise 3D correspondences among the consequent frames. Let us assume that the robot observes a specific point $\mathrm{Pt}$ in the $3 \mathrm{D}$ space, such as $P_{t}=\left[x_{t}, y_{t}, z_{t}\right]^{T}$. In the next time instance $t+1$ the robot undergoes a specific motion with rotation matrix $R_{t+1}^{t}$ and translation vector $T_{t+1}^{t}=\left[T_{x}, T_{y}, T_{z}\right]^{T}$ so the corresponding point $P_{t}$ in now observed as $P_{t+1}=\left[x_{t+1}, y_{t+1}, z_{t+1}\right]^{T}$. The transformation from point $P_{t}$ to $P_{t+1}$ is as follows:

$$
P^{t}=R_{t+1}^{t} \cdot P^{t+1}+T_{t+1}^{t}
$$

Considering 3D registration of all the detected SURF points $\mathbf{P}$, a rigid body transformation could be found that expresses the overall camera (and thus robot) motion estimation in each execution loop. The incremental required rigid body transformation typically should conform with a sum of quadratic differences minimization criterion, resulting to a singular value decomposition (SVD) optimization problem. However, taking into account that errors are introduced mainly stemming from the erroneous SURF feature matching, the depth estimation of the stereo correspondence algorithm and the native camera re-projection error obtained from the stereo pair calibration, an additional outlier rejection method is required to produce refined motion estimations. To this end, a RANSAC outlier detection step has been applied to retain only a subset of inlier points from the set of points $\mathbf{P}$ that satisfy maximum inliers criterion of a plane detection procedure. The retained inlier points are used along the SVD to compute the rovers motion estimation. In the next step, rovers poses and transformations are stored as nodes and edges in the graph respectively, representing the robots trajectory as described in [35]. This graph is further enhanced by registering the generated dense point clouds with respect to the robots pose, as well as by associating the observed features in each node. Having expressed the rovers location estimations as a graph of nodes, a global graph optimization step is applied through g2o [36] optimization when the robot revisits the same location, i.e. observes the same SURF features. The outcome of this procedure is globally consistent robot trajectory which applied the stored 3D point clouds a surface metric map is produced.

\subsection{D reconstruction of underground environment}

Subsurface mapping includes the 3D registration of the detected hyperbolas from the GPR radar-grams to the robots trajectory. To associate underground obtained data from the GPR measurements with robot localization, the robot motion estimation is taken into consideration. During the robots motion, all the A-Scans gathered from the GPR antenna are progressively stored and proportionally distributed to the generated nodes of the graph, assuming constant 
robot velocity. Each A-Scan is thus anchored to a specific node and, as such, it inherits the nodes information, i.e. pose with respect the robots coordinate frame (see Fig. 5). At this step it should be mentioned that given the fact that a B-Scan can be formulated when the GPR antenna performs straight routes, the surface rovers motion estimation is utilized as a criterion for the cut-off and a completion of each radar-gram in accordance to traveled route. Thus, deviations (e.g. $>10^{\circ}$ ) in the robots yaw angle indicate that the robot stopped its straight route and thus all the anchored A-Scans to the pose graph are recalled and concatenated to create a B-Scan. On the constructed B-Scan the above mentioned prep-processing and hyperbola detection procedure is applied to detect the candidate apexes of the buried utilities. From the hyperbola detection algorithm the

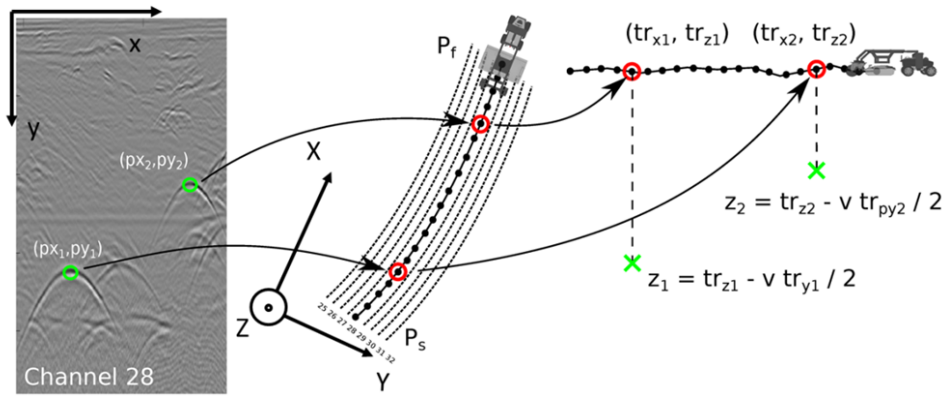

Fig. 5. Transition from the $2 \mathrm{D}$ radar-gram fame (B-Scan) to robot coordinate frame

apex $\left(p x_{i}, p y_{i}\right)$ is computed into the radar-gram where the value $p y_{i}$ in the ordinate (y-axis) denotes the reception time that corresponds to the hyperbola apex in the time window of the recorded signal for an A-Scan, while the value $p x_{i}$ in the abscissa (x-axis) denotes the GPR-antenna covered distance. Therefore, the value $p y_{i}$ should be corrected to indicate the underground depth on which the hyperbola apex belongs to and, this is performed with the following equation:

$$
p z_{i}=\frac{t_{p y i} \cdot u}{2}
$$

where $p z_{i}$ is the actual depth of the apex, $t_{p y i}$ is the reception time and $u$ is the propagation velocity of the medium as illustrated in Fig. 6

\section{$5 \quad$ Perception system assessment}

The evaluation of the smart perception system has been performed both in a simulation as well as in a real environment. In both cases both the hyperbola detection module on its own, as well as the integrated subsurface mapping tool have been evaluated. 


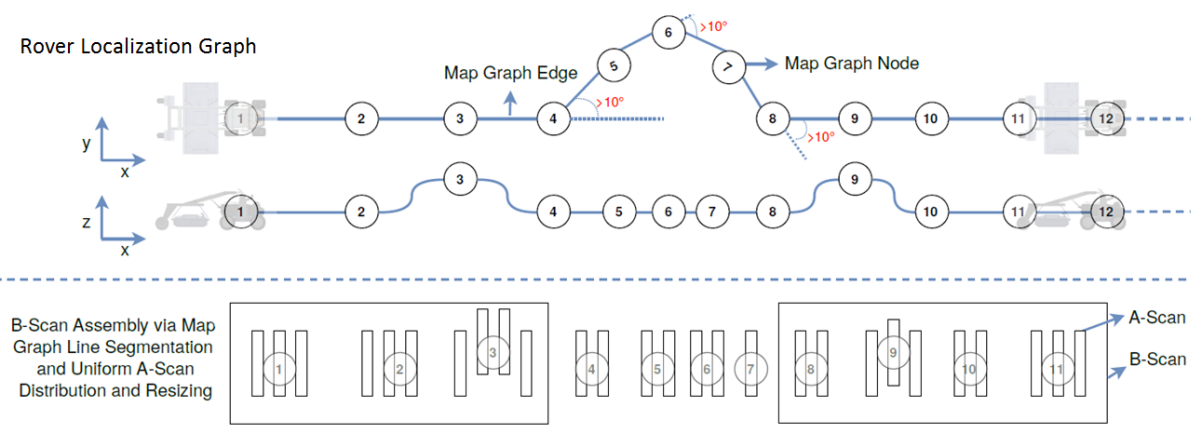

Fig. 6. The pose graph that associates the robots localization and the recorded AScans during robots motion. In the graph it is highlighted how the A-Scan aggregation is performed and the criterion utilized for the B-Scan isolation with respect to the localization graph during rovers travel. Note that node IDs correspond to the aggregated A-Scans and each formulated B-Scan corresponds to the straight line covered by the rover

\subsection{Evaluation in Gazebo simulation}

The performance of the proposed methodology for subsurface mapping has been evaluated through a simulated experiment in the Gazebo 3D robot simulator, the model of which is illustrated Fig. 7(a). A specially designed world was created that, in terms of the surface, consists of mostly flat terrain surrounded on three sides by obstacles, which in combination with a rocky ground texture, offers plenty of features for the visual odometry algorithm. Regarding the subsurface part of the world, five pipes of $20 \mathrm{~cm}$ diameter were added to be used by the subsurface mapping module. In order to enable sonar-based simulated GPR model operation, the space below the ground and between the pipes is left empty. Figure 7 demonstrates the operation of the proposed subsurface mapping system. The existing subsurface utilities, i.e. pipes to be detected, are displayed in the visualization of the robot workspace as dark grey cylinders for reference. As depicted in Fig. 7(b), the robot starts with no information about its environment and gradually constructs a metric representation of it in the form of a point cloud. At the same time, via the simultaneous operation of the subsurface mapping module, the subsurface pipes are detected and visualized through a red pointcloud. This pointcloud corresponds to the apexes of the detected hyperbolas on the B-Scans generated after every straight line as described previously. The detected hyperbola apexes (see Fig. 7(e)) are then processed by the utility mapping module to filter outliers and detect lines corresponding to the pipes. Figure $7(\mathrm{f})$ demonstrates the trajectory followed by the robot as estimated by the visual odometry module in blue, while the groundtruth provided by Gazebo simulation is exhibited with red color. In addition, Fig. $7(\mathrm{~g})$ demonstrates the Euler orientation error of the robot using the Euler angles roll, pitch and yaw. The accuracy of the subsurface mapping depends on the accuracy of the robots localization. The errors in both figures are deemed within acceptable bounds 
for the accurate $3 \mathrm{D}$ registration of the detected hyperbolas, which validates the proposed methodology. However, it should be mentioned that by implementing different localization algorithm the results could be vary accordingly.

\subsection{Evaluation in real test field}

The evaluation of the real system has been performed in a specifically designed and constructed test site. In total, 4 pipes have been placed on the subsurface and among them 3 are metallic and 1 is from plastic (PVC) material. The pipes have been placed in various depths. This ended to multi-variable set-up allowing evaluation of the different aspects of the developed solutions. In the two sides of the test field specific area was kept free of buried utilities to allow surface rover manoeuvring since during the curved motion, the acquired A-Scans are not considered for the construction of the B-Scans. The entire field has been initially measured and the terrain was flattened, so as to allow as smooth as possible antenna operation on the surface of it. The annotation of the buried utilities performed on-the-fly during their installation in order to keep track of the type/size and position of the buried pipes. Thus ground-truth data were kept, however their nominal error is calculated less than $0.02 \mathrm{~cm}$, which has been introduced due to soil displacement effect during the burring phase.

Firstly, the hyperbola detection assessment has been performed on the custom developed test site. The detection percentage of the hyperbolas that are actually correct is $90,1 \%$, yet the recall i.e. the percentage of all annotated hyperbolas that are successfully detected is lower and specifically $75 \%$. The accuracy of the 3D subsurface map is tightly related with the accuracy of the surface rover localization since the data acquired from the GPR antenna are registered to localization pose graph of the surface rover. Therefore, it is essential to firstly quantify the localization error of surface rover. An experiment was performed to test the efficiency and accuracy of the implemented modules. Towards this direction, an experiment was setup in the field-like environment by exploiting pattern specific AprilTags at positions to be detected by the stereo camera and thus used as ground truth estimations. When the rover stands in front of an AprilTag, a custom detector is executed, which provides a ground truth estimation of the pose (position and orientation) of the rover. The ground truth is then compared to the visual odometry provided by rover localization and an Euclidean distance error is computed, as well as an orientation difference error. Those errors are further examined after the rover detects loop closures, by revisiting areas, and optimizing the localization pose graph. Since the point clouds that formulate the map inherit the poses of the localization graph, the error of the optimized map is the overall error of the surface rover localization. The overall positioning error is calculated by considering the Euclidean distance of the position of the visual odometry and the ground truth and found approximately $0.2 \mathrm{~m}$, while the orientation error is calculated by considering the shortest angular distance of the Euler angles (roll, pitch and yaw) of the compared the visual odometry and the ground truth and found to be 0.02 degrees. 
The accuracy of the constructed surface/subsurface map depends mostly on the robots localization accuracy which means that the ability of the system to correctly register the detected utilities on the subsurface map relies on the error of the robots motion estimation. Considering the depth estimation of the buried utilities, there is an uncertainty introduced from the GPR resolution (AScan) resolution of the utilized Stream-C GPR antenna, which of $5 \mathrm{~cm}$. More precisely, in the large scale experiment, the total travelled distance of the robot during this experiment was $60 \mathrm{~m}$. The error in the localization concerning the displacement of the robot after the scanning of the entire field is approximately $0.40 \mathrm{~m}$ which is less than $0.7 \%$ of the total travelled distance. The biggest amount of this deviation is introduced in the $\mathrm{XY}$ plane i.e. the robots motion plane and less deviation has been measured on the $\mathrm{Z}$ axis. The error on the robots orientation was negligible and measured to be less than $1^{\circ}$. The next step of the evaluation comprises the ability of the developed framework to correctly locate the buried pipes. Figure 8 graphically illustrates the 3D subsurface map with the ground truth. Specifically, it is revealed that all the buried pipes in the test site have been successfully mapped in the reconstructed environment. The second row of Figure 8 presents hyperbola detection on indicative B-Scans along the robot traverses. The resulting map has undergone a further outlier detection and DBSCAN step which retained only the most compact descriptions and discarded further erroneous detected apexes from the hyperbola detection step on the BScans. It should be noted that the position of the detected pipes closely resembles the position of the ground-truth with small deviations. The final result shown in Figure 8 closely matches the actual annotated pipes resulting in more than $90 \%$ coverage of the scanned utility network, which means that the $90 \%$ of the buried infrastructures is correctly detected and annotated with this method.

\section{Conclusions}

Summarizing, in this book chapter, a perception system for subsurface mapping using a surface rover and a GPR antenna has been presented. Firstly, a simulation tool for the perception of buried utilities has been developed and has been integrated in the Gazebo environment to be suitable for robotic applications. Then, a utilities detection method on the formulated radargrams from GPR imaging has been constructed. The detection method has been tailored both for the simulation as well as for the real world scenario. Considering the simulation environment image processing and geometrical fitting methods have been used since the acquired data are noise free, while for the real world environment an in depth data preprocessing has been firstly introduced and, then, a method based on HoG features, SVM classification and final fitting approach has been developed to tackle with the readings from the GPR sensor. The perception system both in simulation as well as in the real scenario has been integrated with a surface operating rover where the rover self-localization schema has been integrated with the GPR processing step to create structured subsurface maps, where buried utilities such as pipes are represented in terms of point cloud. The 
smart perception system has been evaluated both in simulation as well as in real scenario and exhibited promising performance since it was proven adequate to construct subsruface maps both is simple as well as in complex real life environments. To this end, the chapter presented a smart application by integrating a real GPR antenna with a surface rover based on technologies fusion from robotics navigation domain combined with radar imaging common in geoscience applications, to provide a perception output for large scale environments facilitating autonomous buried utilities detection.

\section{Acknowledgments}

This work has been supported by the EU Horizon 2020 funded project namely: "RoBot for Autonomous unDerGround trenchless opERations, mapping and navigation" under the grant agreement with no: 731968. 


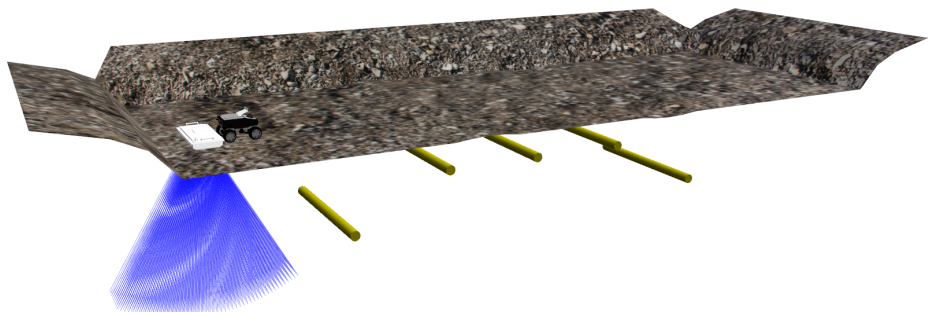

(a)

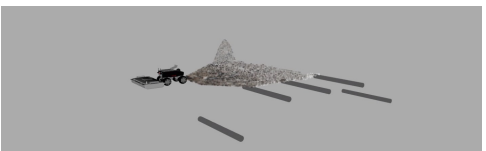

(b)

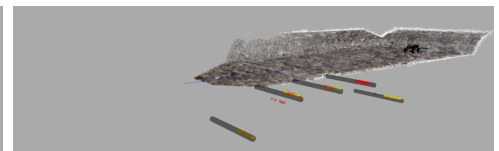

(c)

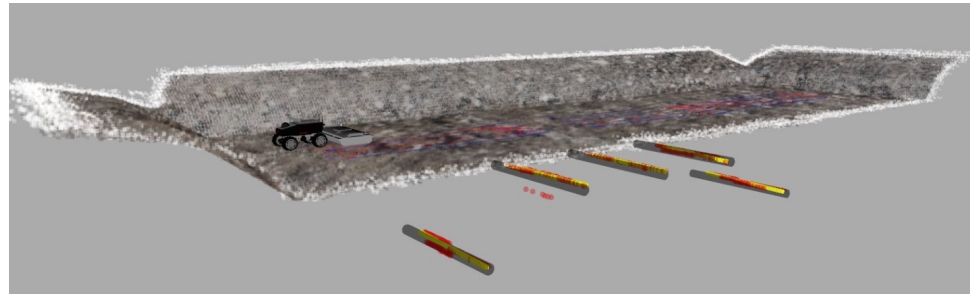

(d)

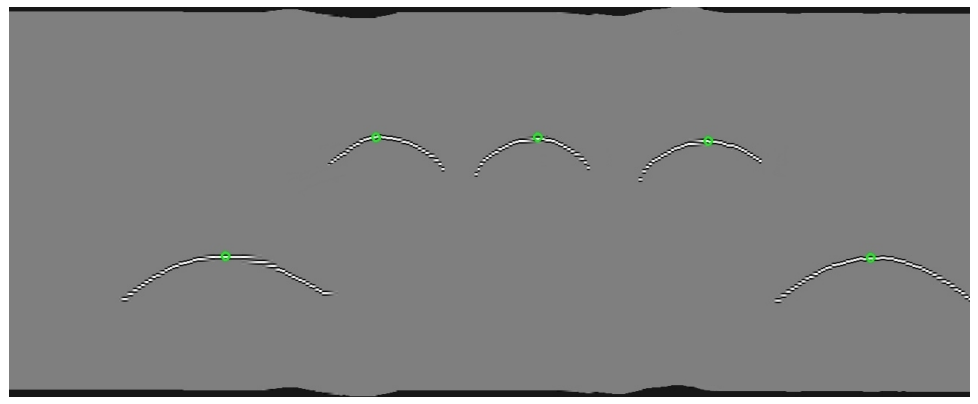

(e)

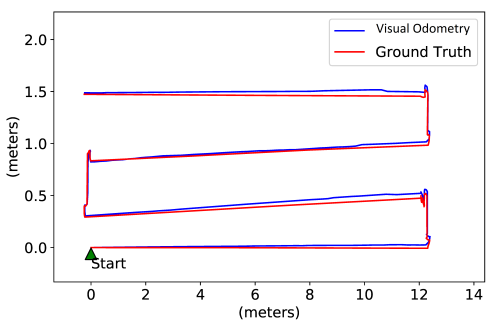

(f)

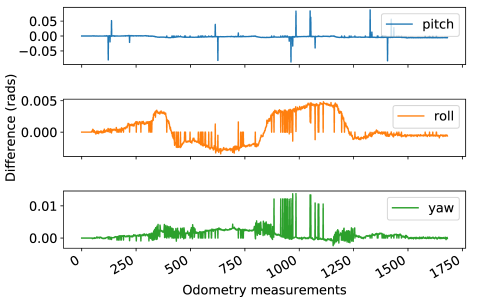

(g)

Fig. 7. Fig. 7(b) - Fig. 7(d) exhibits subsurface mapping at different moments from start to finish. In 7 (d), the full constructed subsurface map is illustrated, where the red dots correspond to the detected pipes and the yellow coloured pipes correspond to the ground truth. The last row refers to the visual odometry error when compared to the Gazebo simulation measurements; left image exhibits the robot trajectory along with the ground truth and right image illustrates the orientation deviation of Euler angles. 


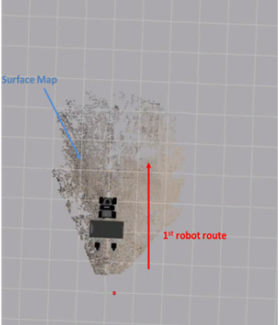

BScan

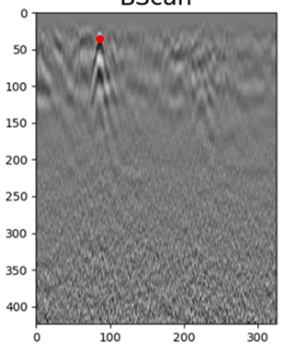

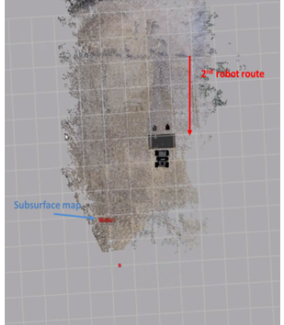

BScan

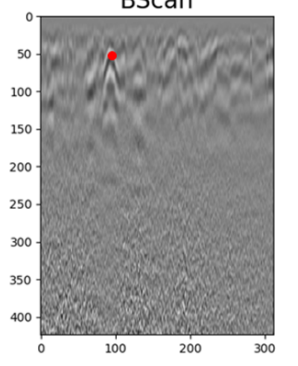

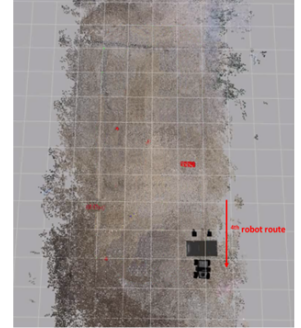

BScan

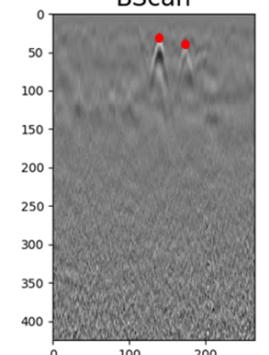

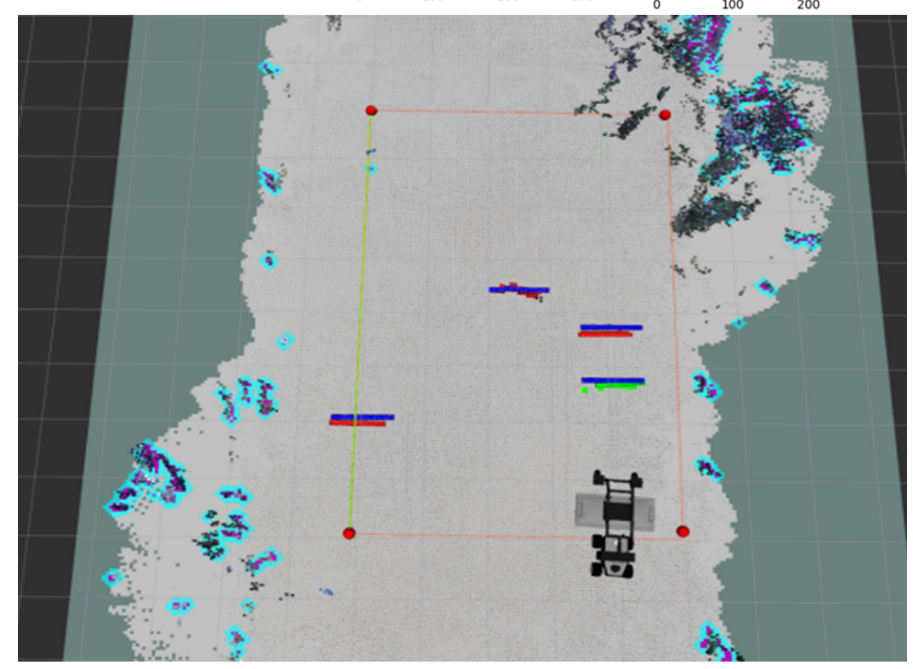

Fig. 8. Evaluation on real dataset. The first row exhibits the progressively built subusurface map, the middle row illustrates the detected hyperbola apexes in three robot passes and the the last row presents constructed subsurface map with the detected pipes. 


\section{Bibliography}

[1] Degenhardt, J.J., Giardino, J.R.: Subsurface investigation of a rock glacier using ground-penetrating radar: Implications for locating stored water on mars. Journal of Geophysical Research: Planets 108(E4) (2003)

[2] De Jongh, R., Ligthart, L., Kaploun, I., Schukin, A., Yarovoy, A.: Design and analysis of new gpr antenna concepts. Tijdschrift-Nederlands Elektronica En Radiogenootschap 64 (1999) 26-32

[3] Feng, X., Sato, M., Zhang, Y., Liu, C., Shi, F., Zhao, Y.: Cmp antenna array gpr and signal-to-clutter ratio improvement. IEEE Geoscience and Remote Sensing Letters 6(1) (2009) 23-27

[4] Kaliampakos, D., Benardos, A.: Underground space development: Setting modern strategies. WIT Transactions on The Built Environment 102 (2008) $1-10$

[5] Aly, M.: Real time detection of lane markers in urban streets. In: Intelligent Vehicles Symposium, 2008 IEEE, IEEE (2008) 7-12

[6] Kouros, G., Psarras, C., Kostavelis, I., Giakoumis, D., Tzovaras, D.: Surface/subsurface mapping with an integrated rover-gpr system: A simulation approach. In: Simulation, Modeling, and Programming for Autonomous Robots (SIMPAR), 2018 IEEE International Conference on, IEEE (2018) $15-22$

[7] Kouros, G., Kostavelis, I., Skartados, E., Giakoumis, D., Tzovaras, D.: $3 \mathrm{~d}$ underground mapping with a mobile robot and a gpr antenna. In: IEEE/RSJ International Conference on Intelligent Robots and Systems (IROS), IEEE (2018) In press

[8] Sandmeier, K.: Reflexw 3.0 manual. Sandmeier Software, Zipser Strabe 1 (2002)

[9] XIE, L.L., JIANG, F.Y., CHANG, W.K.: Forward simulation of the underwater sand based on gprsim software. Geophysical and Geochemical Exploration 6 (2015) 026

[10] Warren, C., Giannopoulos, A., Giannakis, I.: gprmax: Open source software to simulate electromagnetic wave propagation for ground penetrating radar. Computer Physics Communications 209 (2016) 163-170

[11] Annan, A.: Gprhistory, trends, and future developments. Subsurface sensing technologies and applications 3(4) (2002) 253-270

[12] Kim, J.H., Cho, S.J., Yi, M.J.: Removal of ringing noise in gpr data by signal processing. Geosciences Journal 11(1) (2007) 75-81

[13] Torrione, P.A., Throckmorton, C.S., Collins, L.M.: Performance of an adaptive feature-based processor for a wideband ground penetrating radar system. IEEE Transactions on Aerospace and Electronic Systems 42(2) (2006) 644-658

[14] Janning, R., Busche, A., Horváth, T., Schmidt-Thieme, L.: Buried pipe localization using an iterative geometric clustering on gpr data. Artificial Intelligence Review 42(3) (2014) 403-425 
[15] Mertens, L., Persico, R., Matera, L., Lambot, S.: Automated detection of reflection hyperbolas in complex gpr images with no a priori knowledge on the medium. IEEE Transactions on Geoscience and Remote Sensing 54(1) (2016) 580-596

[16] Pasolli, E., Melgani, F., Donelli, M.: Automatic analysis of gpr images: A pattern-recognition approach. IEEE Transactions on Geoscience and Remote Sensing 47(7) (2009) 2206-2217

[17] Illingworth, J., Kittler, J.: A survey of the hough transform. Computer vision, graphics, and image processing 44(1) (1988) 87-116

[18] Falorni, P., Capineri, L., Masotti, L., Pinelli, G.: 3-d radar imaging of buried utilities by features estimation of hyperbolic diffraction patterns in radar scans. In: Ground Penetrating Radar, 2004. GPR 2004. Proceedings of the Tenth International Conference on. Volume 1., IEEE (2004) 403-406

[19] Simi, A., Bracciali, S., Manacorda, G.: Hough transform based automatic pipe detection for array gpr: Algorithm development and on-site tests. In: Radar Conference, 2008. RADAR'08. IEEE, IEEE (2008) 1-6

[20] Dell'Acqua, A., Sarti, A., Tubaro, S., Zanzi, L.: Detection of linear objects in gpr data. Signal Processing 84(4) (2004) 785-799

[21] Borgioli, G., Capineri, L., Falorni, P., Matucci, S., Windsor, C.G.: The detection of buried pipes from time-of-flight radar data. IEEE Transactions on Geoscience and Remote Sensing 46(8) (2008) 2254-2266

[22] Jol, H.M.: Ground penetrating radar theory and applications. elsevier (2008)

[23] Kaur, P., Dana, K.J., Romero, F.A., Gucunski, N.: Automated gpr rebar analysis for robotic bridge deck evaluation. IEEE transactions on cybernetics 46(10) (2016) 2265-2276

[24] La, H.M., Lim, R.S., Basily, B., Gucunski, N., Yi, J., Maher, A., Romero, F.A., Parvardeh, H.: Autonomous robotic system for high-efficiency nondestructive bridge deck inspection and evaluation. In: 2013 IEEE International Conference on Automation Science and Engineering (CASE). (Aug 2013) 1053-1058

[25] Le, T., Gibb, S., Pham, N., La, H., Falk, L., Berendsen, T.: Autonomous robotic system using non-destructive evaluation methods for bridge deck inspection (05 2017)

[26] Williams, R.M., Ray, L.E., Lever, J.H.: Autonomous robotic ground penetrating radar surveys of ice sheets; using machine learning to identify hidden crevasses. In: 2012 IEEE International Conference on Imaging Systems and Techniques Proceedings. (July 2012) 7-12

[27] Ciarletti, V., Clifford, S., Plettemeier, D., Le Gall, A., Herve, Y., Dorizon, S., Quantin-Nataf, C., Benedix, W.S., Schwenzer, S., Pettinelli, E., Heggy, E., Herique, A., Berthelier, J.J., Kofman, W., Vago, J.L., Hamran, S.E.: The WISDOM Radar: Unveiling the Subsurface Beneath the ExoMars Rover and Identifying the Best Locations for Drilling. Astrobiology 17(6-7) (2017) 565 $-584$

[28] Rial, F.I., Pereira, M., Lorenzo, H., Arias, P.: Acquisition and synchronism of gpr and gps data: application on road evaluation. In: Image and Signal 